\title{
Mismatch repair deficiency/microsatellite instability testing as predictive immunotherapy biomarkers-possible diagnostic missteps trusting a single method
}

\author{
Ioanna Gazouli ${ }^{1,2}$, George Zarkavelis ${ }^{1,2}$, Davide Mauri ${ }^{1,2}$, George Pentheroudakis ${ }^{1,2}$ \\ ${ }^{1}$ Department of Medical Oncology, University of Ioannina, Ioannina, Greece; ${ }^{2}$ Society for Study of Clonal Heterogeneity of Neoplasia, EMEKEN, \\ Ioannina, Greece \\ Correspondence to: Ioanna Gazouli. Mpizaniou Street, 8-10, 45221 Ioannina, Greece. Email: ioannagazouli@gmail.com. \\ Provenance: This is an invited article commissioned by the Academic Editor Dr. Jingping Wang (Department of Pathology, The First Affiliated \\ Hospital of Bengbu Medical College, Bengbu Medical College, Bengbu, China). \\ Comment on: Cohen R, Hain E, Buhard O, et al. Association of Primary Resistance to Immune Checkpoint Inhibitors in Metastatic Colorectal Cancer \\ With Misdiagnosis of Microsatellite Instability or Mismatch Repair Deficiency Status. JAMA Oncol 2019;5:551-5.
}

Submitted Sep 18, 2019. Accepted for publication Sep 24, 2019.

doi: $10.21037 /$ atm.2019.09.144

View this article at: http://dx.doi.org/10.21037/atm.2019.09.144

Over the last few years, mismatch repair deficiency (MMR-D)/microsatellite instability (MSI) has been recognized as an indicator of colon carcinomas potentially susceptible to immunotherapy $(1,2)$. To date, two strategies of identifying mismatch repair defects in tumor tissue have been employed: lack of immunohistochemical staining for mismatch repair proteins, in which case the term "mismatch repair deficiency" (MMR-D) is used, and detection of instability of microsatellite DNA tandem repeats in the tumor tissue DNA as compared to germline host DNA, in which case the term "microsatellite instability" (MSI) is used. But do our methods of MMR-D/MSI assessment provide us with infallible results? A recent study (3) reveals that there is a considerable percentage of colon carcinomas falsely identified either as mismatch repair defective or as microsatellite instable, leading to ineffective treatment with immune checkpoint inhibitors.

Immunohistochemical detection of mismatch repair proteins MLH1, MSH2, MSH6, and PMS2 is based on the concept that their absence implies a deficient mismatch repair mechanism in the tumor cells (4). As for MSI, it reflects the accumulation of accidental mutations in the repetitive DNA sequences known as microsatellites, leading to variations of their base pair length that deviate from the cell's germline DNA motif (5). Originally, MSI detection was based on a reference panel of two mononucleotide (BAT26 and BAT25) and three dinucleotide repeat markers (D2S123, D5S346 and D17S250), amplified by polymerase chain reaction (PCR). MSI is defined when 2 or more of the five markers are found instable. This panel was introduced by the National Cancer Institute (NCI) workshop in 1997 (6) and it is still recommended for MSI testing by ESMO guidelines (4). The MSI pentaplex method (7) examines a panel of five poly-A mononucleotide repeat markers (BAT-25, BAT-26, NR-21, NR-24 and NR-27), and it has been shown to be more sensitive and specific compared to the former one, even eliminating the need for comparison to normal tissue DNA. It is currently considered as the gold standard option, due to a reported sensitivity of $95.6 \%$ and a positive predictive value (PPV) of $100 \%$ (8). The pentaplex panel has been suggested to be narrowed down to three of the five markers (BAT26, NR21 and NR27), with a comparable sensitivity (97.4\%) and PPV (96.5\%) (8).

A recent retrospective analysis (3) attempts to challenge the diagnostic validity of immunohistochemistry (IHC) and the MSI pentaplex method. Among the 38 metastatic colorectal cancer (mCRC) patients, all originally identified as either MSI or MMR-D, 3 out of the 5 patients not responding to immune checkpoint inhibitors, were centrally assessed as both mismatch repair proficient and microsatellite stable. Similarly, in an historical cohort of 93 patients diagnosed with MSI or MMR-D mCRC, there were 9 MMR proficient cases, falsely diagnosed 
as microsatellite instable. The above data imply that up to $10 \%$ of patients receiving immunotherapy, based on an MMR-D/MSI diagnosis, could be submitted to an ineffective and costly treatment. Interestingly, 7 of the 12 falsely diagnosed cases in the two examined patient subsets of the trial had been examined only for MSI by PCR, without immunohistochemical verification, while 4 out of 12 cases had been assessed as MSI by PCR, but as MMR proficient by immunohistochemistry. As highlighted by the authors, a notable reason for MSI overdiagnosis lies in rare microsatellite polymorphisms giving the misleading impression of genomic instability. It has also been implied by previous studies (7) that ethnicity related microsatellite variations can be misinterpreted as MSI.

IHC results proved to be false in one case among the 38 examined patients of the study, eventually being classified as MMR proficient by the central review. Falsely negative staining for mismatch repair proteins in the tumor tissue can be due to inappropriate tissue handling and fixation (9). In addition, all four MMR proteins have to be examined, given that in the absence of one, its partner protein can still form a stable and functional heterodimer with a substitute repair protein unrecognized by the antibodies (10).

An alternative method of MSI detection, applied in the study, employs HT17, a highly conserved gene encoding the heat shock protein (HSP) 110, as a microsatellite marker (3). Specifically, a dominant-negative form of HSP110 (HSP110DE9) can be expressed due to large, biallelic deletions in the T[17] intron repeat of HSP110 in tumor DNA, thus impairing the stress resistance mechanisms of cancer cells (11). Indeed, the HT17 method has previously been found to have better sensitivity (98.4\% vs. 95\%) and similar specificity (99.7\% for both) in detecting hypermutated tumors, compared with the pentaplex panel (12). Among four samples examined by Cohen et al., recognized as MMR-D by IHC but as MSS by PCR molecular testing, two were found to exhibit MSI when HT17 testing was applied (3).
Next generation sequencing (NGS) is a high-throughput method enabling concurrent determination of the tumor mutational burden (TMB) and specific gene mutations considered of significant prognostic or predictive value in metastatic colon cancer (e.g., KRAS, BRAF). NGSbased genomic instability detection may prove to be a more reliable method of determining eligibility for immunotherapy, as it can accurately recognize true deviations from DNA repeated motifs by actually decoding DNA sequences, rather than comparing microsatellites length. Reported sensitivity and specificity NGS rates are up to $92 \%$ and $100 \%$ respectively, among colon carcinomas immunohistochemically recognized as MMR deficient (13). Although currently employed only in specialized centers, it is regarded as a promising future option for assessing the mutational status of cancer cells with higher accuracy (4). Moreover, NGS holds potential for the detection of hypermutated tumors in the absence of MMR-D/MSI (such as POLE mutated tumors).

In summary, recognizing tumors with impaired DNA repair mechanisms or accumulation of mutations has been used for selecting patients eligible for immunotherapy, due to their high antigenicity. Nonetheless, our methods of detecting MMR deficiency or MSI are not to be regarded as unquestionable; in fact, they have a considerable chance of providing us with misleading results. A combinatorial approach of the already available methods, IHC and MSI detection, could possibly improve the diagnostic accuracy. A recent position paper by ESMO recommends serial testing by immunohistochemical staining for the four mismatch repair proteins (MLH1, MSH2, MSH6, PMS2), and consecutively by MSI-PCR, preferentially by the five poly-A mononucleotide panel, in order to eliminate ambiguous results (see Table 1) (4). More modern approaches as HT17 gene examination and NGS may prove to serve as more efficient biomarkers, once they become more widely available among diagnostic centers and hospitals. 
Table 1 Summarizing the current ESMO recommendations for MMR-D/MSI assessment, as stated by Luchini et al. [see (4)]

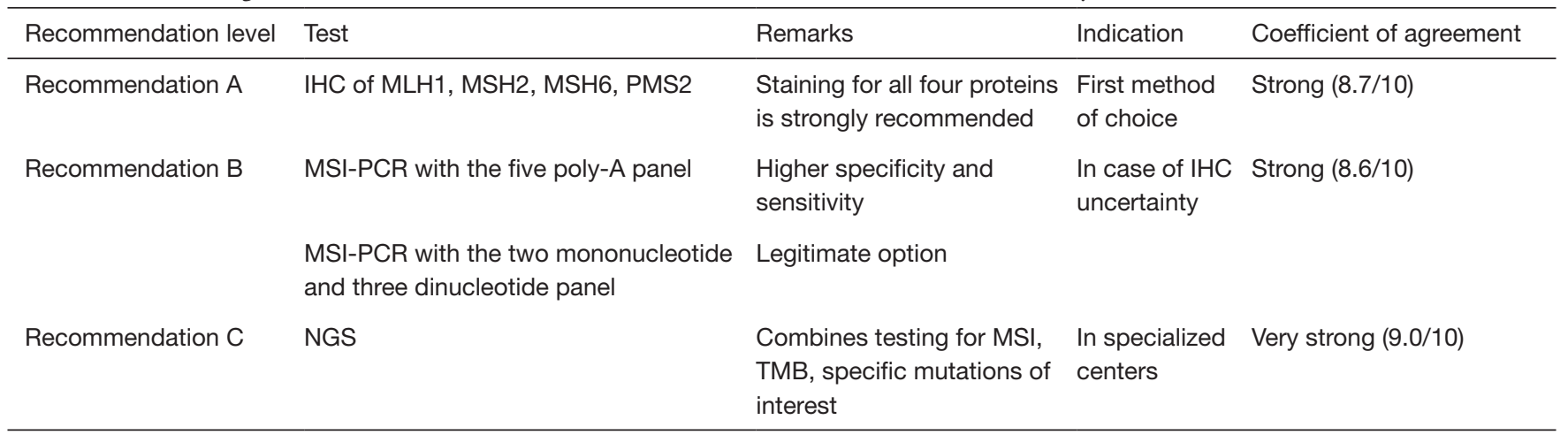

IHC, immunohistochemistry; NGS, next generation sequencing; MMR-D/MSI, mismatch repair deficiency/microsatellite instability; PCR, polymerase chain reaction; TMB, tumor mutational burden.

\section{Acknowledgments}

None.

\section{Footnote}

Conflicts of Interest: The authors have no conflicts of interest to declare.

Ethical Statement: The authors are accountable for all aspects of the work in ensuring that questions related to the accuracy or integrity of any part of the work are appropriately investigated and resolved.

\section{References}

1. Le DT, Uram JN, Wang H, et al. PD-1 Blockade in Tumors with Mismatch-Repair Deficiency. N Engl J Med 2015;372:2509-20.

2. Overman MJ, Lonardi S, Wong KYM, et al. Durable Clinical Benefit With Nivolumab Plus Ipilimumab in DNA Mismatch Repair-Deficient/Microsatellite Instability-High Metastatic Colorectal Cancer. J Clin Oncol 2018;36:773-9.

3. Cohen R, Hain E, Buhard O, et al. Association of Primary Resistance to Immune Checkpoint Inhibitors in Metastatic Colorectal Cancer With Misdiagnosis of Microsatellite Instability or Mismatch Repair Deficiency Status. JAMA Oncol 2019;5:551-5.

4. Luchini C, Bibeau F, Ligtenberg MJL, et al. ESMO recommendations on microsatellite instability testing for immunotherapy in cancer, and its relationship with PD-1/ PD-L1 expression and tumour mutational burden: a systematic review-based approach. Ann Oncol 2019. [Epub ahead of print].

5. Sinicrope FA, Sargent DJ. Molecular pathways: microsatellite instability in colorectal cancer: prognostic, predictive, and therapeutic implications. Clin Cancer Res 2012;18:1506-12.

6. Boland CR, Thibodeau SN, Hamilton SR, et al. A National Cancer Institute Workshop on Microsatellite Instability for cancer detection and familial predisposition: development of international criteria for the determination of microsatellite instability in colorectal cancer. Cancer Res 1998;58:5248-57.

7. Suraweera N, Duval A, Reperant M, et al. Evaluation of tumor microsatellite instability using five quasimonomorphic mononucleotide repeats and pentaplex PCR. Gastroenterology 2002;123:1804-11.

8. Goel A, Nagasaka T, Hamelin R, et al. An optimized pentaplex PCR for detecting DNA mismatch repairdeficient colorectal cancers. PLoS One 2010;5:e9393.

9. Engel KB, Moore HM. Effects of preanalytical variables on the detection of proteins by immunohistochemistry in formalin-fixed, paraffin-embedded tissue. Arch Pathol Lab Med 2011;135:537-43.

10. Pearlman R, Markow M, Knight D, et al. Two-stain immunohistochemical screening for Lynch syndrome in colorectal cancer may fail to detect mismatch repair deficiency. Mod Pathol 2018;31:1891-900.

11. Collura A, Lagrange A, Svrcek M, et al. Patients with colorectal tumors with microsatellite instability and large deletions in HSP110 T17 have improved response to 5-fluorouracil-based chemotherapy. Gastroenterology 2014;146:401-11.e1.

12. Buhard O, Lagrange A, Guilloux A, et al. HSP110 T17 
Page 4 of 4

simplifies and improves the microsatellite instability testing in patients with colorectal cancer. J Med Genet 2016;53:377-84.

13. Nowak JA, Yurgelun MB, Bruce JL, et al. Detection of

Cite this article as: Gazouli I, Zarkavelis G, Mauri D, Pentheroudakis G. Mismatch repair deficiency/microsatellite instability testing as predictive immunotherapy biomarkerspossible diagnostic missteps trusting a single method. Ann Transl Med 2019;7(Suppl 8):S329. doi: 10.21037/atm.2019.09.144

\section{Gazouli et al. MMR-D/MSI as biomarkers-diagnostic missteps}

Mismatch Repair Deficiency and Microsatellite Instability in Colorectal Adenocarcinoma by Targeted NextGeneration Sequencing. J Mol Diagn 2017;19:84-91. 